\title{
Effectiveness and feasibility of early physical rehabilitation programs for geriatric hospitalized patients: a systematic review
}

\author{
Nienke M Kosse ${ }^{1 *}$, Alisa L Dutmer ${ }^{1}$, Lena Dasenbrock², Jürgen M Bauer ${ }^{2}$ and Claudine JC Lamoth ${ }^{1}$
}

\begin{abstract}
Background: Old adults admitted to the hospital are at severe risk of functional loss during hospitalization. Early in-hospital physical rehabilitation programs appear to prevent functional loss in geriatric patients. The first aim of this review was to investigate the effect of early physical rehabilitation programs on physical functioning among geriatric patients acutely admitted to the hospital. The second aim was to evaluate the feasibility of early physical rehabilitation programs.
\end{abstract}

Methods: Two searches, one for physical functioning and one for feasibility, were conducted in PubMed, CINAHL, and EMBASE. Additional studies were identified through reference and citation tracking. To be included articles had to report on in-hospital early physical rehabilitation of patients aged 65 years and older with an outcome measure of physical functioning. Studies were excluded when the treatment was performed on specialized units other than geriatric units. Randomized controlled trials were included to examine the effect of early physical rehabilitation on physical functioning, length of stay and discharge destination. To investigate feasibility also non randomized controlled trials were added.

Results: Fifteen articles, reporting on 13 studies, described the effect on physical functioning. The early physical rehabilitation programs were classified in multidisciplinary programs with an exercise component and usual care with an exercise component. Multidisciplinary programs focussed more on facilitating discharge home and independent $\mathrm{ADL}$, whereas exercise programs aimed at improving functional outcomes. At time of discharge patients who had participated in a multidisciplinary program or exercise program improved more on physical functional tests and were less likely to be discharged to a nursing home compared to patients receiving only usual care. In addition, multidisciplinary programs reduced the length of hospital stay significantly. Follow-up interventions improved physical functioning after discharge. The feasibility search yielded four articles. The feasibility results showed that early physical rehabilitation for acutely hospitalized old adults was safe. Adherence rates differed between studies and the recruitment of patients was sometimes challenging.

Conclusions: Early physical rehabilitation care for acutely hospitalized old adults leads to functional benefits and can be safely executed. Further research is needed to specifically quantify the physical component in early physical rehabilitation programs.

Keywords: Acute care, Rehabilitation, Hospital, Aged, Functional outcomes, Feasibility, ADL, Physical performance

\footnotetext{
* Correspondence: n.m.kosse@umcg.nl

${ }^{1}$ University of Groningen, University Medical Center Groningen, Center for

Human Movement Sciences, Groningen, The Netherlands

Full list of author information is available at the end of the article
} 


\section{Background}

The rapidly growing population of old adults in Western countries has become a major concern for health care systems. Due to a poorer health status, old adults consume a disproportionate amount of medical care. In some European countries, more than $40 \%$ of patients admitted to the hospital for an overnight stay are aged 65 years and older, while their total share of the population is less than $20 \%$ [1].

Unfortunately, old adults admitted to the hospital are at severe risk of functional decline, both during hospitalization and after discharge [2,3]. A number of studies found that approximately $33 \%$ of the patients have severe functional deterioration at time of discharge compared to their status before hospital admission [3-5]. For patients 90 years or older this number even increases to $63 \%$ [4]. Functional decline during and after hospital stay has shown to be an important risk factor for nursing home placement $[6,7]$.

The decline in functional capacity seems to be partly the result of the hospitalization itself, unrelated to diagnostic or therapeutic interventions. Older patients have decreased physiological and functional reserves that make them more vulnerable to the effects of bed rest and decrease in dietary intake, which both are highly prevalent during hospitalization. Due to immobilisation, muscle strength and aerobic capacity tend to decline rapidly. After only ten days of bed rest healthy old adults lose $12-14 \%$ of both their $\mathrm{VO}_{2 \max }$ and lower extremity muscle strength [8]. Without any voluntary muscle contractions muscle strength can even decrease by $5 \%$ per day [2].

Altogether, functional decline is a common problem that is significantly associated with negative outcomes such as institutionalization, re-hospitalization and subsequent mortality [3]. The primary focus of hospital care is treating acute and chronic illnesses. A physical rehabilitation intervention that may preserve physical function is often not part of the treatment. To preclude a rapid decline in physical function it is important that hospital programs are also directed explicitly towards activating the older patient early after hospital admission. Early physical rehabilitation might help to prevent decline in physical functioning arising from immobility and prolonged bed rest [9].

Over the years, several multidisciplinary and exercise types of early rehabilitation interventions have been studied. Previous studies showed that early rehabilitation programs improved both patient (e.g. physical functioning) and hospital outcomes (e.g. reducing costs) for acute ill geriatric patients $[10,11]$. However, an important issue not yet addressed in the current literature is the feasibility of in-hospital early exercise programs for acute geriatric patients. To start an early physical rehabilitation program, knowledge of which patient population benefit from the program is required. Furthermore, it is important to know if there will be adverse events during early physical rehabilitation programs in terms of falls or other injuries and what the adherence rate of the patients will be during the treatment sessions. Therefore, the first aim of this review is to evaluate the effects of early physical rehabilitation programs on physical functioning of geriatric patients acutely admitted to a hospital. In this review early physical rehabilitation in acutely ill patients refers to physical therapy, occupational therapy, and physical exercises initiated immediately upon achieving physiologic stability and continued throughout the hospital stay. Such activities start within 1 or 2 days after hospitalization. The second aim of the present review is to evaluate the feasibility of early physical rehabilitation programs in the hospitalized geriatric patients.

\section{Method}

\section{Search strategy}

A systematic literature search was conducted in three electronic databases, PubMed, CINAHL, and EMBASE in August 2013. Keywords used to perform the search were: "aged" or "elderly" or "geriatric", "hospital" or "hospitalized", "exercise" or "rehabilitation", "ADL" or "physical functioning" or "mobility" or "physical performance", and "acute" or "acutely". The articles included were randomized controlled trials (RCT), written in English, including participants aged 65 years or older who were acutely admitted to the hospital. The interventions investigated in the articles needed to include a physical exercise part with a physical functioning measure as outcome. Studies were excluded if the included patients required treatment on a specialized unit other than an acute geriatric unit or when the evaluated intervention aimed at a specific disorder or surgical process. Articles about the feasibility of early physical rehabilitation of inpatients were retrieved by adding the keywords "feasibility" or "feasible" or "adherence rate" or "safety" to the keywords mentioned above. The articles about feasibility had the same in and exclusion criteria as the articles addressing physical functioning with the exception that also non randomized controlled trials were included. For the inclusion process title and abstract were examined and when necessary the full article was obtained and read. Additional studies were identified through reference and citation tracking. Two reviewers independently screened title, abstract and full text. Disagreement about inclusion of articles was resolved by discussion and consensus between the two reviewers.

\section{Data extraction and analysis}

Data were extracted against pre-defined categories by two researchers. The data compiled from the studies included information on: study design, characteristics of 
participants and setting, the intervention and control group treatment, time of assessment, ADL, physical performance, length of stay and discharge destination. Furthermore, the feasibility outcomes were the ability to enrol patients into the rehabilitation program, and the adherence rate and safety of the patients during the therapy sessions. The information extracted from the articles was organized into tables and systematically compared.

\section{Methodological quality}

The methodological quality of the included RCTs was assessed using the Delphi scale [12]. The Delphi scale is a quality assessment tool for RCTs and has shown to be valid and reliable [13]. It consists of 9 different criteria which can be scored positive, negative, or unclear ("yes", "no", and "don't know"). One point was given for each "yes" and zero points for each "no" or "don't know", the total quality sore ranged from 0 (low quality) to 9 (high quality).

\section{Results}

\section{Selected studies}

The literature search for physical functioning yielded a total of 772 papers (Figure 1). After removing 765 articles based on title and abstract, 9 articles were qualified for full text reading. Four articles were removed after full text reading and ten articles were added after reference checking. The remaining 15 articles, describing 13 studies, were included to this review. The search for feasibility studies yielded 50 papers. After removing 47 articles by title, abstract and full text reading and adding one article after reference checking, four studies were included for the feasibility assessment. The article of Laver et al. [14] was included in both the physical functioning and the feasibility section. PRISMA guidelines were followed in this systematic review [15].

\section{Methodological quality}

In Table 1 the quality scores on the Delphi Scale for the different RCT studies are reported. Total quality scores ranged from 3 to 7 with a median score of 5 . The methodological quality was moderate for most studies. Randomization methods and eligibility criteria were clearly defined in all 13 studies. The studies scored particularly low on blinding the assessor, the care provider and the participant. The Delphi scores were good for the concealed treatment allocation, the similarity of the intervention and control groups at baseline and the clarity of the specified eligibility criteria.

\section{Inclusion criteria and patient characteristics}

Table 2 summarizes the characteristics of patients, study settings, early physical rehabilitation programs and outcomes of the included RCT studies in this review. The mean age for patients admitted to acute care in the hospital for a general medical condition varied between 78 and 86 years old. The most common reasons for admission were cardiac problems, respiratory problems, gastrointestinal problems, neurological problems, infections and injuries caused by a fall. The living situation of the patients before they were admitted to the hospital varied, patients came from nursing homes and other types of institutionalised care or from the community where they lived alone or with family. However, the

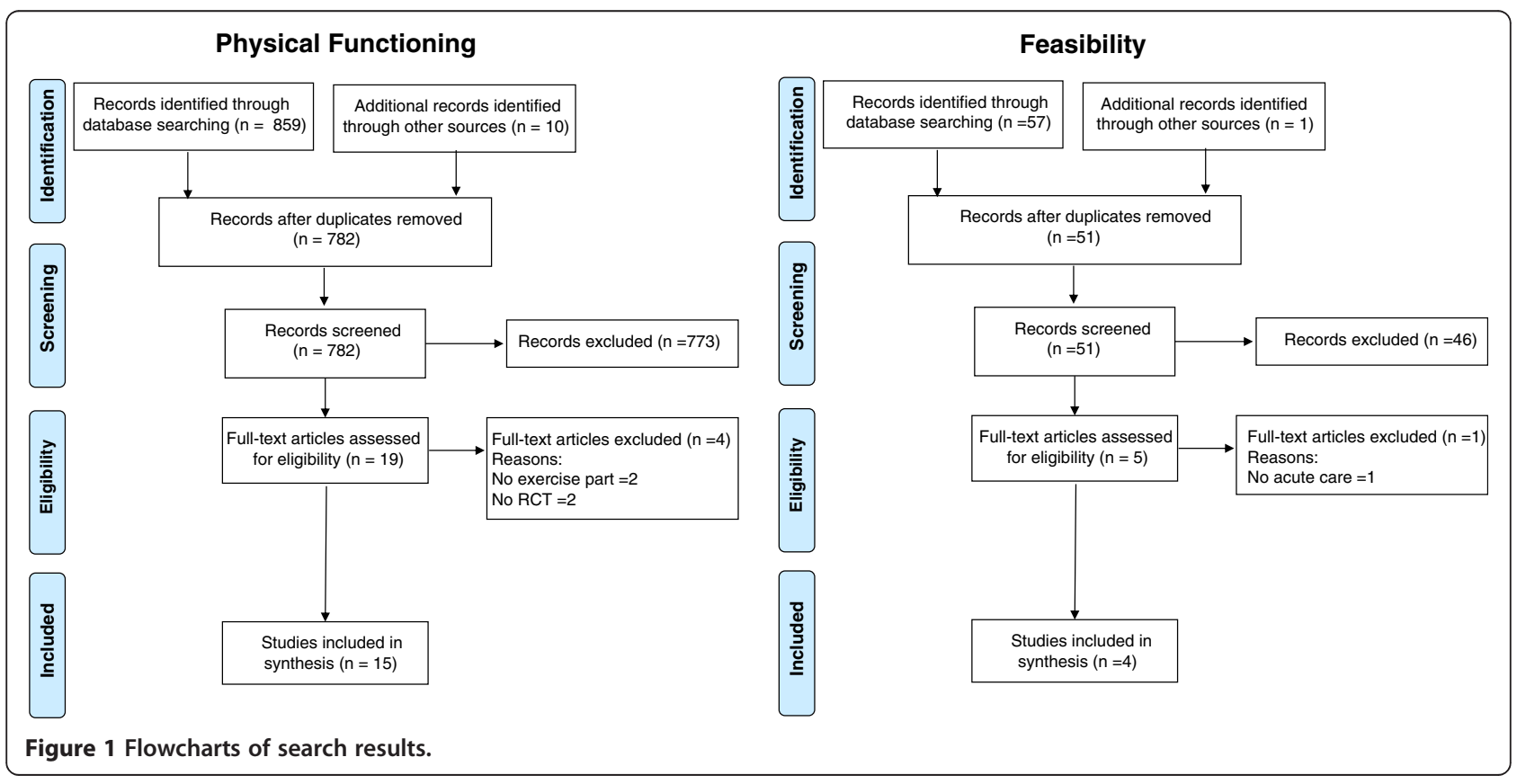


Table 1 Methodological quality scores on the Delphi scale for each RCT study

\begin{tabular}{|c|c|c|c|c|c|c|c|c|c|c|}
\hline Study & Randomized & $\begin{array}{l}\text { Treatment } \\
\text { allocation } \\
\text { concealed }\end{array}$ & $\begin{array}{c}\text { Groups } \\
\text { similar at } \\
\text { baseline }\end{array}$ & $\begin{array}{c}\text { Eligibility } \\
\text { criteria } \\
\text { specified }\end{array}$ & $\begin{array}{c}\text { Outcome } \\
\text { assessor } \\
\text { blinded }\end{array}$ & $\begin{array}{c}\text { Care } \\
\text { provider } \\
\text { blinded }\end{array}$ & $\begin{array}{l}\text { Patient } \\
\text { blinded }\end{array}$ & $\begin{array}{l}\text { Variability } \\
\text { measures }\end{array}$ & $\begin{array}{l}\text { Intention-to-treat } \\
\text { analysis }\end{array}$ & $\begin{array}{l}\text { Total } \\
(\max 9)\end{array}$ \\
\hline Abizanda [16] & 1 & 1 & 0 & 1 & 1 & 1 & 1 & 1 & 0 & 7 \\
\hline Asplund [17] & 1 & 1 & 1 & 1 & 0 & 0 & 0 & 0 & 0 & 4 \\
\hline Blanc-Bisson [18] & 1 & 0 & 1 & 1 & 0 & 0 & 0 & 1 & 1 & 5 \\
\hline Counsell [19] & 1 & 1 & 1 & 1 & 0 & 0 & 0 & 1 & 1 & 6 \\
\hline Courtney $[20,21]$ & 1 & 1 & 1 & 1 & 1 & 0 & 0 & 1 & 1 & 7 \\
\hline De Morton [22] & 1 & 0 & 0 & 1 & 0 & 0 & 1 & 1 & 1 & 5 \\
\hline Jones [23] & 1 & 1 & 0 & 1 & 0 & 0 & 0 & 1 & 1 & 5 \\
\hline Landefeld [24] & 1 & 0 & 1 & 1 & 0 & 0 & 0 & 0 & 0 & 3 \\
\hline Laver [14] & 1 & 1 & 1 & 1 & 1 & 0 & 0 & 1 & 1 & 7 \\
\hline Nikolaus [25] & 1 & 1 & 1 & 1 & 1 & 0 & 0 & 1 & 0 & 6 \\
\hline Saltvedt $[26,27]$ & 1 & 1 & 1 & 1 & 0 & 0 & 0 & 1 & 0 & 5 \\
\hline Siebens [28] & 1 & 1 & 1 & 1 & 0 & 0 & 0 & 0 & 1 & 5 \\
\hline Slaets [29] & 1 & 1 & 1 & 1 & 0 & 0 & 0 & 0 & 0 & 4 \\
\hline
\end{tabular}

studies did not include all patients in the intervention, reasons for exclusion were medical instability [18,22,23], need for specialized care $[17,19,22,24]$, living in nursing homes $[19,20,22,23,25,26,28]$, small survival chance or need for palliative care $[18,22,23,25,28]$, and being diagnosed with an illness causing functional impairment $[18,23,28]$. Overall, there was a great heterogeneity among the participants between the different studies.

\section{Setting and intervention}

Table 2 gives an overview of the settings and interventions of each included randomized controlled trial. The studies were performed on acute geriatric units, geriatric units and medical units of university hospitals, city hospitals and acute care hospitals. Early physical rehabilitation programs could be divided into two categories, (1) multidisciplinary programs with an exercise component and (2) usual care with an additional exercise program. In the review we refer to these categories as multidisciplinary programs and exercise programs, respectively. The main aim of the multidisciplinary programs was to maintain or obtain independent ADL and encourage returning home. Multidisciplinary intervention teams usually consisted of a geriatrician, (geriatric) nurses, social workers, physical therapists and occupational therapists $[17,19,24,26,29]$. The specific exercise component of the multidisciplinary intervention studies is hardly described, and information on intensity, duration, and frequency of exercises is often lacking. Usual care with an additional exercise program was provided in eight studies $[14,16,18,20,22,23,25,28]$. The patients in the exercise programs were supervised by allied health assistants, a physiotherapist or an occupational therapist. Patients performed exercises five times a week up to twice daily.
The aim of the exercise programs was predominantly to improving functional outcomes by training strength, mobility, and balance. Strength exercises were progressed by increasing the number of sets and repetitions and walking exercises were progressed in intensity (from slow to moderate pace) or duration (from 5 to $30 \mathrm{mi}$ nutes). In one study an interactive video gaming program was used to exercise. A Nintendo Wii fit group trained $25 \mathrm{~min} /$ day, 5 days/week under supervision of a physical therapist [14]. Some exercise programs supported participants to continue exercise after discharge $[20,25,28]$. This was achieved by educational materials, by (two or more regular) encouraging phone calls and home visits $[20,28]$, or by a follow-up treatment, twice a week up to twice a day, including physical and occupational therapy [25]. The control groups of the studies generally received usual care according to the general routines of the hospital they were admitted to.

\section{Physical functioning}

All included studies provided at least one outcome measure related to physical functioning. Most studies used measures of activities of daily living (ADL) and physical performance to describe physical functioning. Additional information about length of stay and discharge destination were also documented.

\section{Activities of daily living}

The included studies gained information on patient's ADL by conducting interviews and (self-administered) questionnaires. Table 3 gives on overview of the used instruments to measure (I)ADL. Eleven of the thirteen studies reported the effects of their intervention on (I)ADL at time of hospital discharge [14,16-19,21-25,29]. 
Table 2 Setting and study characteristics physical functioning

\begin{tabular}{|c|c|c|c|c|c|c|c|c|}
\hline \multirow[t]{2}{*}{ Study } & \multirow[t]{2}{*}{ Population \& Setting } & \multirow[t]{2}{*}{ Intervention } & \multicolumn{6}{|l|}{ Time of assessment } \\
\hline & & & & (I)ADL & $\begin{array}{l}\text { Physical } \\
\text { Performance }\end{array}$ & $\begin{array}{l}\text { Mortality } \\
(\%)\end{array}$ & $\begin{array}{l}\text { LOS } \\
\text { (days) }\end{array}$ & $\begin{array}{l}\text { Discharge } \\
\text { ICF (\%) }\end{array}$ \\
\hline \multicolumn{9}{|c|}{ Multidisciplinary care with an exercise component } \\
\hline \multirow[t]{6}{*}{ Asplund $2000[17]$} & Intervention $(n=190)$ & \multirow{3}{*}{$\begin{array}{l}\text { Multidisciplinary team, with physical and occupational } \\
\text { therapy. Discharge planning and early rehabilitation. }\end{array}$} & T0 Admission & $\mathrm{BI} \geq 19$ points & \multirow[t]{6}{*}{ NA } & T1 4.2 & \multirow[t]{3}{*}{$5.9^{*}$} & T1 11.6 \\
\hline & Mean age 80.9 years & & T1 Discharge & T0 52\% & & T2 11.1 & & T2 11.6 \\
\hline & $58 \%$ female, AGU, UH & & T2 3 months post-discharge & T2 44\% & & & & \\
\hline & Control $(n=223)$ & \multirow[t]{3}{*}{ General medical unit care } & & $\mathrm{Bl} \geq 19$ points & & T1 2.7 & $7.3^{*}$ & T1 19.3 \\
\hline & Mean age 81.0 years & & & T0 44\% & & T2 7.6 & & T2 18.4 \\
\hline & $63 \%$ female, MU, UH & & & T2 43\% & & & & \\
\hline \multirow[t]{13}{*}{ Counsell 2000 [19] } & Intervention $(n=767) \dagger$ & \multirow{6}{*}{$\begin{array}{l}\text { Multidisciplinary team, with daily assessment of } \\
\text { physical functioning and protocols to improve } \\
\text { self-care and mobility. Early discharge planning. }\end{array}$} & T0 2 weeks pre-admission & ADL decline & PPME score & T1 2.7 & 6.1 & T1 12.9 \\
\hline & Mean age 80 years & & T1 Discharge & T1 30\% & \multirow[t]{5}{*}{$\mathrm{T} 15.6^{*}$} & T2 9.0 & & T2 10.3 \\
\hline & \multirow[t]{4}{*}{$60 \%$ female, $\mathrm{CH}$} & & T2 1 months post-discharge & T2 27\% & & T3 15.9 & & T3 8.9 \\
\hline & & & T3 3 months post-discharge & T3 26\% & & T4 22.6 & & T4 7.5 \\
\hline & & & T4 6 months post-discharge & T4 22\% & & T5 31.4 & & T5 6.7 \\
\hline & & & T5 1 year post-discharge & T5 25\% & & & & \\
\hline & Control $(n=764) \dagger$ & \multirow[t]{7}{*}{ Usual physician and nursing staff care } & & ADL decline & PPME score & T1 3.7 & 6.3 & T1 15.6 \\
\hline & Mean age 79 years & & & T1 34\% & \multirow[t]{6}{*}{$\mathrm{T} 15.0^{*}$} & T2 11.3 & & T2 10.1 \\
\hline & \multirow[t]{5}{*}{$61 \%$ female, $\mathrm{CH}$} & & & T2 29\% & & & & \\
\hline & & & & T3 26\% & & Т3 17.4 & & T3 7.2 \\
\hline & & & & T4 30\% & & & & \\
\hline & & & & T5 30\% & & T4 22.5 & & T4 8.0 \\
\hline & & & & & & T5 29.2 & & T5 7.3 \\
\hline \multirow[t]{8}{*}{ Landefeld 1995 [24] } & Intervention $(\mathrm{n}=327)$ & \multirow{8}{*}{$\begin{array}{l}\text { Multidisciplinary program, with daily assessment } \\
\text { of physical functioning and protocols to improve } \\
\text { self-care and mobility }\end{array}$} & T0 Admission & ADL score: & \multirow[t]{8}{*}{ NA } & T1 7.3 & \multirow[t]{8}{*}{7.3} & $\mathrm{~T} 15.8^{*}$ \\
\hline & Mean age 80.2 years & & T1 Discharge & T0 3.0 & & T2 20.8 & & T2 $13.1^{*}$ \\
\hline & $68 \%$ female, $\mathrm{MU}, \mathrm{CH}$ & & T2 3 months post-discharge & $\mathrm{T} 13.6^{*}$ & & & & \\
\hline & & & & $\mathrm{T} 24.0$ & & & & \\
\hline & & & & IADL score: & & & & \\
\hline & & & & T0 2.8 & & & & \\
\hline & & & & $\mathrm{T} 13.3^{*}$ & & & & \\
\hline & & & & T2 3.9 & & & & \\
\hline
\end{tabular}


Table 2 Setting and study characteristics physical functioning (Continued)

\begin{tabular}{|c|c|c|c|c|c|c|c|c|}
\hline & Control $(n=324)$ & \multirow{6}{*}{$\begin{array}{l}\text { Usual care services provided by } \\
\text { physicians and nurses }\end{array}$} & & \multirow{2}{*}{\multicolumn{2}{|c|}{$\begin{array}{l}\text { ADL score: } \\
\text { T0 } 3.0\end{array}$}} & $\mathrm{~T} 17.4$ & 8.3 & T1 $11.7^{*}$ \\
\hline & Mean age 80.1 years & & & & & T2 19.8 & & T2 $18.8^{*}$ \\
\hline & $65 \%$ female, $\mathrm{MU}, \mathrm{CH}$ & & & \multicolumn{2}{|l|}{$\mathrm{T} 23.8$} & & & \\
\hline & & & & \multicolumn{2}{|l|}{ IADL score: } & & & \\
\hline & & & & \multicolumn{2}{|l|}{ T0 2.8} & & & \\
\hline & & & & \multicolumn{2}{|l|}{ T2 3.8} & & & \\
\hline \multirow[t]{16}{*}{ Saltvedt $2002[26,27]$} & Intervention $(\mathrm{n}=127) \dagger$ & \multirow{8}{*}{$\begin{array}{l}\text { Interdisciplinary program to prevent } \\
\text { complications, with early mobilization, } \\
\text { rehabilitation and discharge planning }\end{array}$} & T0 Admission & ADL dependence & \multirow[t]{8}{*}{ NA } & $\mathrm{T} 111.8^{*}$ & $15^{*}$ & NA \\
\hline & Mean age 81.8 years & & T1 3 months post-discharge & T1 21\% & & $\mathrm{T} 326.8^{*}$ & & \\
\hline & $81 \%$ female, GU, UH & & T2 6 months post-discharge & T2 13\% & & & & \\
\hline & & & T3 1 year post-discharge & \multicolumn{2}{|l|}{ T3 25\% } & & & \\
\hline & & & & \multicolumn{2}{|l|}{ IADL dependence } & & & \\
\hline & & & & \multicolumn{2}{|l|}{$\mathrm{T} 146 \%$} & & & \\
\hline & & & & \multicolumn{2}{|l|}{ T2 44\% } & & & \\
\hline & & & & \multicolumn{2}{|l|}{ T3 45\% } & & & \\
\hline & Control $(n=127) \dagger$ & \multirow[t]{8}{*}{ Usual care } & & \multicolumn{2}{|l|}{ ADL dependence } & $\mathrm{T} 127.6^{*}$ & $7^{*}$ & \\
\hline & Mean age 82.4 years & & & \multicolumn{2}{|l|}{$\mathrm{T} 112 \%$} & T2 $33.9^{*}$ & & \\
\hline & $84 \%$ female, MU, UH & & & \multicolumn{2}{|l|}{ T2 13\% } & & & \\
\hline & & & & \multicolumn{2}{|l|}{ Т3 23\% } & & & \\
\hline & & & & \multicolumn{2}{|l|}{ IADL dependence } & & & \\
\hline & & & & \multicolumn{2}{|l|}{ T1 39\% } & & & \\
\hline & & & & \multicolumn{2}{|l|}{ T2 40\% } & & & \\
\hline & & & & \multicolumn{2}{|l|}{ T3 44\% } & & & \\
\hline \multirow[t]{6}{*}{ Slaets 1997 [29] } & $\begin{array}{l}\text { Intervention } \\
(n=140)\end{array}$ & \multirow{3}{*}{$\begin{array}{l}\text { Multidisciplinary program added to the } \\
\text { usual care. Geriatrician, physiotherapist } \\
\text { and liaison nurse obtained optimal ADL } \\
\text { and mobility in } 2 \text { hours training a day. }\end{array}$} & T0 Admission & Improved ADL & $\begin{array}{l}\text { Improved } \\
\text { mobility }\end{array}$ & NA & $19.7^{*}$ & T2 $18^{*}$ \\
\hline & Mean age 82.5 years & & T1 Discharge & T1 61\% ${ }^{*}$ & T1 48\% ${ }^{*}$ & & & \\
\hline & $67 \%$ female $, \mathrm{MU}, \mathrm{CH}$ & & T2 1 year post-discharge & & & & & \\
\hline & Control $(n=97)$ & \multirow[t]{3}{*}{$\begin{array}{l}\text { Usual care: services provided by physicians } \\
\text { and nurses. }\end{array}$} & & Improved ADL & $\begin{array}{l}\text { Improved } \\
\text { mobility }\end{array}$ & & $24.8^{*}$ & $\mathrm{~T} 227^{*}$ \\
\hline & Mean age 83.2 years & & & T1 46\% ${ }^{*}$ & T1 44\% ${ }^{*}$ & & & \\
\hline & 75\% female, MU, CH & & & & & & & \\
\hline
\end{tabular}


Table 2 Setting and study characteristics physical functioning (Continued)

\begin{tabular}{|c|c|c|c|c|c|c|c|c|}
\hline \multicolumn{3}{|c|}{ Usual care programs with an exercise intervention } & \multirow{3}{*}{$\begin{array}{l}\text { T0 Admission } \\
\text { T1 Discharge }\end{array}$} & \multirow{3}{*}{$\begin{array}{l}55.6 \% \text { improved } \\
\geq 10 \mathrm{Bl} \text { points }\end{array}$} & \multirow[b]{2}{*}{ NA } & \multirow{3}{*}{ T1 7.6} & \multirow{3}{*}{9.1} & \multirow[b]{2}{*}{ NA } \\
\hline \multirow[t]{6}{*}{ Abinzanda 2011 [16] } & Intervention $(n=198)$ & \multirow{3}{*}{$\begin{array}{l}\text { Conventional treatment plus occupational } \\
\text { therapy: } 5 \text { days per week, } 30 \text { - } 45 \text { min a day }\end{array}$} & & & & & & \\
\hline & Mean age 83.7 years & & & & & & & \\
\hline & $56.6 \%$ female, AGU, UH & & & & & & & \\
\hline & Control $(n=202)$ & \multirow{3}{*}{$\begin{array}{l}\text { Conventional treatment: medical treatment, } \\
\text { nursing care, physical therapy, and social } \\
\text { assistance according with the usual practice } \\
\text { of the unit. }\end{array}$} & & \multirow{3}{*}{$\begin{array}{l}36.7 \% \text { improved } \\
\geq 10 \mathrm{Bl} \text { points }\end{array}$} & & T1 11.9 & 8.7 & \\
\hline & Mean age 83.3 years & & & & & & & \\
\hline & $56.9 \%$ female, AGU, UH & & & & & & & \\
\hline \multirow[t]{8}{*}{ Blanc-Bisson 2008 [18] } & Intervention $(\mathrm{n}=38)$ & \multirow{4}{*}{$\begin{array}{l}\text { Usual care plus early intensive physical therapy } \\
\text { program: start day } 1-2 \text {, strength training twice } \\
\text { a day half an hour, } 5 \text { days a week until T1 }\end{array}$} & T0 Admission & Mean Katz index & NA & T1 5.3 & $\mathrm{~T} 1$ & NA \\
\hline & Mean age 85.5 years & & T1 Clinical stability & T0 6.7 & & T2 7.9 & 12.6 & \\
\hline & \multirow[t]{2}{*}{$66 \%$ female, AGU, UH } & & \multirow{6}{*}{$\begin{array}{l}\text { T2 } 1 \text { month after clinical } \\
\text { stability }\end{array}$} & T1 5.3 & & & & \\
\hline & & & & $\mathrm{T} 24.5$ & & & & \\
\hline & Control $(n=38)$ & \multirow{4}{*}{$\begin{array}{l}\text { Usual care: transferred to arm-chair asap. Start } \\
\text { day 3-6 walking } 3 \text { times a week with human } \\
\text { help or without assistance. Physical therapy } \\
\text { at home for } 1 \text { month }\end{array}$} & & Mean Katz index & & T1 5.3 & $\mathrm{~T} 1$ & \\
\hline & Mean age 85.4 years & & & T0 6.0 & & $\mathrm{~T} 15.3$ & & \\
\hline & \multirow[t]{2}{*}{ 79\% female, AGU, UH } & & & $\mathrm{T} 14.7$ & & & & \\
\hline & & & & T2 3.0 & & & & \\
\hline \multirow[t]{15}{*}{ Courtney 2009 [20,21] } & Intervention $(n=64) \dagger$ & \multirow{15}{*}{$\begin{array}{l}\text { Individual exercise program and nursing } \\
\text { visits, performed daily or several times a } \\
\text { week. The intervention continued at home } \\
\text { with home visits and regular telephone } \\
\text { follow-up by a nurse. }\end{array}$} & T0 Admission & ADL: Mean score & WIQ distance* & T1 1.6 & 4.6 & NA \\
\hline & Mean age 78.1 years & & T1 4 weeks post-discharge & index of $A D L^{*}$ & T0 23.54 & T2 3.1 & & \\
\hline & \multirow[t]{13}{*}{$62 \%$ female, $\mathrm{MU}, \mathrm{CH}$} & & T2 12 weeks post-discharge & T0 0.36 & T1 53.62 & T3 3.1 & & \\
\hline & & & T3 24 weeks post-discharge & T1 0.07 & T2 54.83 & & & \\
\hline & & & & T2 0.18 & T3 62.89 & & & \\
\hline & & & & T3 0.16 & WIQ speed ${ }^{*}$ & & & \\
\hline & & & & $\begin{array}{l}\text { IADL: Mean } \\
\text { IADL scale }\end{array}$ & T0 16.21 & & & \\
\hline & & & & T0 2.16 & T1 41.30 & & & \\
\hline & & & & $\mathrm{T} 11.47$ & T2 44.62 & & & \\
\hline & & & & T2 1.27 & T3 48.56 & & & \\
\hline & & & & Т3 1.13 & WIQ stairs ${ }^{*}$ & & & \\
\hline & & & & & T0 27.70 & & & \\
\hline & & & & & T1 46.73 & & & \\
\hline & & & & & T2 51.23 & & & \\
\hline & & & & & T3 57.20 & & & \\
\hline
\end{tabular}


Table 2 Setting and study characteristics physical functioning (Continued)

\begin{tabular}{|c|c|c|c|c|c|c|c|c|}
\hline & Control $(n=64)+$ & \multirow{15}{*}{$\begin{array}{l}\text { Routine care, discharge planning and } \\
\text { rehabilitation advice. If necessary, } \\
\text { in-home follow-up. }\end{array}$} & & $\begin{array}{l}A D L \text { : Mean score } \\
\text { index of } A D L^{*}\end{array}$ & WIQ distance ${ }^{*}$ & T1 4.7 & \multirow[t]{15}{*}{4.7} & \\
\hline & Mean age 79.4 years & & & T0 0.35 & T0 20.22 & T2 4.7 & & \\
\hline & $63 \%$ female, $\mathrm{MU}, \mathrm{CH}$ & & & T1 0.69 & T1 28.90 & T3 4.7 & & \\
\hline & & & & T2 0.75 & T2 21.59 & & & \\
\hline & & & & T3 1.27 & T3 19.93 & & & \\
\hline & & & & $\begin{array}{l}\text { IADL: Mean } \\
\text { IADL scale }\end{array}$ & WIQ speed ${ }^{*}$ & & & \\
\hline & & & & T0 2.62 & T0 14.43 & & & \\
\hline & & & & T1 3.29 & T1 22.09 & & & \\
\hline & & & & T2 3.56 & T2 17.89 & & & \\
\hline & & & & T3 4.33 & T3 16.58 & & & \\
\hline & & & & & WIQ stairs ${ }^{*}$ & & & \\
\hline & & & & & T0 24.12 & & & \\
\hline & & & & & T1 26.06 & & & \\
\hline & & & & & T2 24.40 & & & \\
\hline & & & & & T3 22.18 & & & \\
\hline \multirow[t]{12}{*}{ De Morton 2007 [22] } & Intervention $(n=110) \dagger$ & \multirow{6}{*}{$\begin{array}{l}\text { Usual care plus an individual exercise } \\
\text { program. Twice daily, } 5 \text { days a week, } \\
\text { for } 20-30 \text { minutes. }\end{array}$} & T0 Admission & Mean BI: & Mean TUG (s): & T1 1.8 & \multirow[t]{6}{*}{5.0} & \multirow[t]{6}{*}{ T1 18.2} \\
\hline & Mean age 78 years & & T1 Discharge & T0 66 & T0 35 & & & \\
\hline & $54 \%$ female, MU, ACH & & & T1 79 & T1 36 & & & \\
\hline & & & & & Mean FAC: & & & \\
\hline & & & & & T0 4.0 & & & \\
\hline & & & & & T1 4.8 & & & \\
\hline & Control $(n=126) \dagger$ & \multirow{6}{*}{\multicolumn{2}{|c|}{$\begin{array}{l}\text { Usual care: daily medical assessment, } 24 \text { hour } \\
\text { nursing assistance, and allied health service } \\
\text { on referral from medical, nursing or other } \\
\text { allied health staff. }\end{array}$}} & Mean BI: & Mean TUG (s): & T1 1.6 & 6.0 & \multirow[t]{6}{*}{ T1 20.6} \\
\hline & Mean age 80 years & & & T0 68 & T0 30 & & & \\
\hline & $56 \%$ female, MU, ACH & & & T1 75 & T1 26 & & & \\
\hline & & & & & Mean FAC: & & & \\
\hline & & & & & T0 3.9 & & & \\
\hline & & & & & T1 4.7 & & & \\
\hline
\end{tabular}


Table 2 Setting and study characteristics physical functioning (Continued)

\begin{tabular}{|c|c|c|c|c|c|c|c|c|}
\hline \multirow[t]{6}{*}{ Jones 2006 [23] } & Intervention $(n=80) \dagger$ & \multirow{3}{*}{$\begin{array}{l}\text { Usual care plus an individual exercise } \\
\text { program Twice daily for approximately } \\
30 \text { minutes. }\end{array}$} & \multirow[t]{6}{*}{ T0 Admission T1 Discharge } & \multirow{3}{*}{$\begin{array}{l}\text { Mean change } \\
\text { mBl: } 11 \text { points }\end{array}$} & \multirow{3}{*}{$\begin{array}{l}\text { Mean change } \\
\text { TUG: } 5.4 \mathrm{sec}^{*}\end{array}$} & \multirow{3}{*}{$\mathrm{T} 15.0$} & \multirow[t]{3}{*}{9} & \multirow[t]{3}{*}{ T1 32.5} \\
\hline & Mean age 81.9 years & & & & & & & \\
\hline & 54\% female, MU, ACH & & & & & & & \\
\hline & Control $(n=80)+$ & \multirow{3}{*}{$\begin{array}{l}\text { Usual care: medical, nursing and allied health } \\
\text { intervention and discharge planning consistent } \\
\text { with the patient's diagnosis and resources } \\
\text { available on the acute general medical wards. }\end{array}$} & & \multirow{3}{*}{$\begin{array}{l}\text { Mean change } \\
\text { mBl: } 9 \text { points }\end{array}$} & \multirow{3}{*}{$\begin{array}{l}\text { Mean change } \\
\text { TUG: } 1.2 \mathrm{sec}^{*}\end{array}$} & \multirow[t]{3}{*}{ T1 2.5} & \multirow[t]{3}{*}{11} & \multirow[t]{3}{*}{ T1 51.3} \\
\hline & Mean age 82.9 years & & & & & & & \\
\hline & $61 \%$ female, MU, ACH & & & & & & & \\
\hline \multirow[t]{6}{*}{ Laver 2012 [14] } & Intervention ( $\mathrm{n}=22)$ & \multirow{3}{*}{$\begin{array}{l}\text { Individual interactive video game program } \\
\text { (Wii Fit) } 25 \mathrm{~min} / \text { day, } 5 \text { days/week supervised } \\
\text { by a physiotherapist }\end{array}$} & T0 Admission & IADL & TUG & \multirow[t]{3}{*}{$\mathrm{T} 10$} & \multirow[t]{3}{*}{12.3} & \multirow[t]{3}{*}{ NA } \\
\hline & Mean age 85.2 years & & T1 Discharge & T0 181 & T0 38 & & & \\
\hline & $86 \%$ female $\mathrm{GU}, \mathrm{ACH}$ & & & T1 205 & T1 28 & & & \\
\hline & Control (n=22) & \multirow{3}{*}{$\begin{array}{l}\text { Conventional physiotherapy, matching the } \\
\text { patients abilities and treatment needs } \\
25 \text { min/day, } 5 \text { days/week }\end{array}$} & & IADL & TUG & \multirow[t]{3}{*}{$\mathrm{T} 10$} & \multirow[t]{3}{*}{14.95} & \multirow[t]{3}{*}{ NA } \\
\hline & Mean age 84.6 years & & & T0 141 & T0 35 & & & \\
\hline & 73\% female GU, ACH & & & T1 190 & T1 29 & & & \\
\hline \multirow[t]{21}{*}{ Nikolaus 1999 [25] } & $\begin{array}{l}\text { Intervention with } \\
\text { follow-up }(n=181) \dagger\end{array}$ & \multirow{7}{*}{$\begin{array}{l}\text { In-hospital and post-discharge follow-up } \\
\text { treatment by an interdisciplinary team. } \\
\text { Physical or occupational therapy twice a } \\
\text { week up to twice a day for } 30 \text { min }\end{array}$} & T0 Admission & Mean BI score: & \multirow[t]{21}{*}{ NA } & \multirow[t]{7}{*}{ T2 18.2} & \multirow[t]{7}{*}{$33.5^{*}$} & $\mathrm{~T} 14.4^{*}$ \\
\hline & Mean age 81.4 years & & T1 Discharge & T0 71.0 & & & & T2 16.6 \\
\hline & Female $73.4 \%, \mathrm{GU}, \mathrm{UH}$ & & T2 1 year post-discharge & T1 91.8 & & & & \\
\hline & & & & T2 81.2 & & & & \\
\hline & & & & Mean LB score: & & & & \\
\hline & & & & T1 5.7 & & & & \\
\hline & & & & T2 $5.6^{*}$ & & & & \\
\hline & $\begin{array}{l}\text { Intervention without } \\
\text { follow-up }(n=179) \dagger\end{array}$ & $\begin{array}{l}\text { In-hospital treatment by an interdisciplinary } \\
\text { team, followed by usual care at home }\end{array}$ & & Mean score Bl: & & T2 16.8 & $40.7^{*}$ & $\mathrm{~T} 17.3^{*}$ \\
\hline & Mean age 81.4 years & & & T0 71.0 & & & & T2 18.4 \\
\hline & Female $73.4 \%$, GU, UH & & & T1 92.6 & & & & \\
\hline & & & & T2 82.3 & & & & \\
\hline & & & & Mean LB score: & & & & \\
\hline & & & & T1 5.5 & & & & \\
\hline & & & & T2 $4.1^{*}$ & & & & \\
\hline & Control $(n=185) \dagger$ & Usual care in hospital & & Mean score Bl: & & T2 17.3 & $42.7^{*}$ & $\mathrm{~T} 18.1^{*}$ \\
\hline & Mean age 81.4 years & & & T0 71.0 & & & & T2 22.7 \\
\hline & Female $73.4 \%$, GU, UH & & & T1 91.1 & & & & \\
\hline & & & & T2 80.9 & & & & \\
\hline & & & & Mean LB score: & & & & \\
\hline & & & & T1 5.5 & & & & \\
\hline & & & & T2 4.3 & & & & \\
\hline
\end{tabular}

Mean age 81.4 years

Female 73.4\%, GU, UH

Control $(n=185)$

T2 4.3 
Table 2 Setting and study characteristics physical functioning (Continued)

Siebens 2000 [28] Intervention $(\mathrm{n}=149)+$ Hospital-based exercise program twice a day.

Encouragement to continue the program at hom

T0 2 weeks pre-admission

Mean number of

Independent

Mean age 78.2 years

T1 1 month post-discharge

independent IADL walking

$62 \%$ female, $\mathrm{ACH}$
T0 $59.7 \%$

Control $(n=151)+\quad$ Usual care

$\mathrm{T} 15.1^{*}$

T1 $64.2 \%$

Mean number of Independent T1 6.6

10.5

Mean age 78.5 years

ADL independence walking

$59 \%$ female, ACH

T0 5.3

T0 50.3\%

T1 $4.6^{*}$

T1 65.5\%

$\uparrow=$ included only community dwelling old adults; ADL= Activities of Daily Living; IADL= Instrumental Activities of Daily Living; ICF=Intramural Care Facility; AGU=Acute Geriatric Unit; GU=Geriatric Unit; MU=Medical Unit; $\mathrm{UH}=$ University Hospital; $\mathrm{CH}=\mathrm{City}$ Hospital; $\mathrm{ACH}=\mathrm{Acute}$ Care Hospital; * $=$ significant $(\mathrm{p}>0.05)$; PPME=Physical Performance and Mobility Examination; WIQ=Walking Impairment Questionnaire; TUG=Timed Up and Go; FAC=Functional ambulation classification; NA=not available. 
Table 3 Overview of the instruments used to measure (I)ADL and physical performance

\begin{tabular}{|c|c|c|c|c|c|}
\hline \multirow[t]{2}{*}{ Instrument } & \multirow[t]{2}{*}{ Study } & \multirow[t]{2}{*}{ Assessment } & \multicolumn{3}{|c|}{ Score } \\
\hline & & & Min & Max & Interpretation \\
\hline \multirow[t]{3}{*}{ Katz index/ADL index } & Courtney [20] & $\begin{array}{l}\text { Ability to perform: Bathing, eating, dressing, continence, transfer to toilets } \\
\text { and locomotion }\end{array}$ & 0 & 6 & 0 independent - 6 dependent \\
\hline & Bizon-Blanc [18] & & 0 & 12 & 0 independent - 12 dependent \\
\hline & Counsell and Landefeld $[19,24]$ & $\begin{array}{l}\text { Ability to perform: Bathing, dressing, using the toilet, moving from a bed to } \\
\text { a chair, and eating }\end{array}$ & 0 & 5 & 0 independent - 5 dependent \\
\hline \multirow[t]{2}{*}{ (modified) Barthel index } & Asplund and Salvedt $[17,26]$ & \multirow{2}{*}{$\begin{array}{l}\text { Feeding, urinary and faecal continence, personal toilet, dressing, toilet use, } \\
\text { transferring, walking outdoors, climbing stairs and bathing }\end{array}$} & 0 & 20 & 1 dependent - 20 independent \\
\hline & $\begin{array}{l}\text { Abizanda, Jones, De Morton, } \\
\text { Nicolaus }[16,22,23,25]\end{array}$ & & 0 & 100 & 0 dependent - 100 independent \\
\hline \multirow[t]{2}{*}{ Lawton index/IADL index } & Nikolaus and Salvedt $[25,26]$ & $\begin{array}{l}\text { Ability to use telephone, shopping, food preparation, housekeeping, laundry, mode } \\
\text { of transportation, responsibility for medication and ability to handle finances }\end{array}$ & 0 & 8 & 0 dependent - 8 independent \\
\hline & $\begin{array}{l}\text { Counsell, Courtney, Landefeld } \\
\text { and Siebens }[19,20,24,28]\end{array}$ & $\begin{array}{l}\text { Ability to use telephone, shopping, food preparation, housekeeping, mode of } \\
\text { transportation, responsibility for medication and ability to handle finances }\end{array}$ & 0 & 7 & 0 dependent - 7 independent \\
\hline \multirow[t]{2}{*}{ Timed IADL } & \multirow[t]{2}{*}{ Laver [14] } & The time needed to complete tasks addressing five IADL domains: & & & \multirow{2}{*}{$\begin{array}{l}\text { Lower scores indicates } \\
\text { greater ability }\end{array}$} \\
\hline & & (1) communication, (2) finance, (3) cooking, (4) shopping, and (5) medicine. & & & \\
\hline SIVIS dependency scales & Sleats [29] & $\begin{array}{l}\text { SIVIS independency scale: } 20 \text { questions relating to orientation, communication, } \\
\text { mobility, transfers, ADL, continence, catheter use, and decubitus }\end{array}$ & & & NA \\
\hline $\begin{array}{l}\text { Functional Independence } \\
\text { Measure (FIM) }\end{array}$ & Laver and Siebens $[14,28]$ & $\begin{array}{l}\text { Measures the level of a patient's disability and indicates how much } \\
\text { assistance is required for the individual to carry out activities of daily } \\
\text { living: Eating, Grooming, Bathing, Upper and lower body dressing, Toileting, } \\
\text { Bladder and bowel management, Bed to chair transfer, Toilet and shower } \\
\text { transfer, Locomotion, Stairs, Cognitive comprehension, Expression, Social } \\
\text { interaction, Problem solving, Memory }\end{array}$ & 18 & 126 & 18 dependent - 126 independent \\
\hline $\begin{array}{l}\text { Walking Impairment } \\
\text { Questionnaire (WIQ) }\end{array}$ & Courtney [20] & Walking distance, walking speed and climbing stairs & 0 & 100 & $\begin{array}{l}\text { Higher scores indicates } \\
\text { greater ability }\end{array}$ \\
\hline Timed Up and Go (TUG) & $\begin{array}{l}\text { Jones, Laver, } \\
\text { De Morton }[14,22,23]\end{array}$ & $\begin{array}{l}\text { Time taken for the patient to rise from a chair, walk } 3 \mathrm{~m} \text {, turn and walk } \\
\text { back to the chair }\end{array}$ & & & $\begin{array}{l}\text { Lower scores indicates } \\
\text { greater ability }\end{array}$ \\
\hline Functional Ambulation Classification & De Morton [22] & Ability to ambulate over a 10 foot distance and $4 \mathrm{~m}$ length of foam & 0 & 6 & 0 dependent - 6 independent \\
\hline Physical activity scale & Siebens [28] & $\begin{array}{l}\text { Questionnaire about walking 1/4 mile, walking up } 10 \text { steps, } \\
\text { crouching/kneeling, lifting/carrying } 10 \mathrm{lbs}\end{array}$ & & & NA \\
\hline Mobility & Counsell [19] & $\begin{array}{l}\text { Walking to a table, walking inside the house, walking a block, walking } \\
\text { uphill or upstairs, and running a short distance }\end{array}$ & & & NA \\
\hline $\begin{array}{l}\text { Physical Performance and } \\
\text { Mobility Examination }\end{array}$ & Counsell [19] & $\begin{array}{l}\text { Bed mobility, transfer skills, multiple stands, standing balance, step up } \\
\text { one step and timed } 6 \mathrm{~m} \text { walk }\end{array}$ & 0 & 6 & 0 dependent -6 independent \\
\hline Short Physical Performance Battery & Laver [14] & $\begin{array}{l}\text { Three standing balance measures (tandem, semi-tandem, and side-by-side } \\
\text { stands), five continuous chair stands, and a 2.44-meter walk. }\end{array}$ & 0 & 12 & 0 dependent -12 independent \\
\hline Modified Berg Balance Scale & Laver [14] & & & & NA \\
\hline
\end{tabular}


Two multidisciplinary programs found that patients in exercise group compared to the patients in usual care group significantly improved more and worsened less in the number of basic ADL activities they were able to perform [24,29]. The other studies did not find a significant group difference in ADL at time of discharge. However, one study with an exercise intervention found a significant interaction effect between group and admission scores on the modified Barthel index meaning that patients with a low admission score who received an exercise intervention showed greater improvement in ADL than those patients who received only usual care [23]. Two studies reported a ceiling effect for the Barthel index and a floor effect for the Katz index [22,23]. Remaining studies did not found a significant difference on ADL between early physical rehabilitation programs and usual care (see Table 2).

Of the eight studies that reported follow-up data on (I)ADL [17-20,24-26,28] only the three studies with an exercise intervention that provided patients with follow-up treatment after hospital discharge reported larger improvements in the exercise group than in the usual care group after 1, 6 and 12 months $[20,25,28]$. One study that did not provide patients with follow-up treatment also found a positive effect of the intervention on ADL at 3 months post-discharge. However, this study included patients who died by assigning them a score of zero, whereas the mortality rate was higher in the usual care group than the multidisciplinary group [26].

\section{Physical performance}

Seven studies reported measures of physical performance; Table 3 gives an overview of the used measurement instruments $[14,19,20,22,23,28,29]$. Three (2 multidisciplinary programs and 1 exercise programs) of the five studies describing physical performance at time of admission and discharge found that the intervention groups improved more or declined less than the usual care groups $[19,23,29]$. However, for one study the difference in the Timed Up and Go (TUG) was not significant after adjusting for confounders such as patient characteristics, admission modified Barthel Index, comorbidity, and mental scores [23].

For the TUG a floor effect was found. In one study, almost $40 \%$ of the patients were physically unable to perform the TUG at both admission and discharge [23], whereas in another study $23 \%$ of all patients were unable to perform the TUG at admission to the hospital [22].

Two studies reported follow-up results on physical performance, but the results are ambiguous [20,28]. One study [28] reported no significant difference between the patients in the exercise group and in the usual care group in the change in physical performance scores obtained 2 weeks before admission and one month after discharge. Conversely, the other study [20] reports that the exercise group had greater improvement over time (up to 24 weeks after discharge) in walking distance, walking speed and stair climbing. Similar contradicting results were found on more general outcome measures of physical functioning, provided by a health questionnaire that contained physical well-being and physical activity. No group differences were found for the National Health Survey Physical Activity Scale at one month follow-up [28], while the other study found that the exercise group scored higher than the usual care group on physical health related quality of life at 4, 12, and 24 weeks after discharge [21]. In short, follow-up results on physical performance are contradictory. Nevertheless, at time of discharge most studies show a greater improvement for patients in the multidisciplinary and exercise group than for the patients in the usual care group.

\section{Length of stay and discharge destination}

All included randomized controlled trials reported about the length of stay, which varied between the 4.7 days and 42.7 days. Four studies (three multidisciplinary programs) reported a significant difference shorter length of stay of the exercise group than for the usual care group $[17,25,27,29]$.

From the seven studies $[17,19,22-25,29]$ that reported on discharge destination, five studies found that a higher proportion of patients in the early physical rehabilitation group than in the usual care group were discharged home, instead of being transferred to additional (subacute) hospital treatment or to institutionalized care, however, results were significant in three studies (Table 2). Two multidisciplinary programs found that respectively $14 \%$ and $18 \%$ of the patients in the multidisciplinary group were discharged to a long-term care institution opposed to $22 \%$ and $27 \%$ of usual care patients $[24,29]$. The third study, an exercise intervention, included only patients who had lived at home before admission and found that $4 \%$ of the patients in the exercise group and $8 \%$ of the patients in the usual care group were discharged to longterm care institutions [25].

\section{Feasibility}

Feasibility of early physical rehabilitation programs, was explicitly assessed in four studies (Table 4) comparing usual care with an exercise intervention program [14,30-32]. In addition included randomized controlled trails with an exercise program reported on enrolment $(n=3)$, adherence rate $(n=2)$ and adverse events $(n=5)$, while multidisciplinary studies reported on enrolment $(n=3)$ only.

Subjects enrolled in the studies explicitly assessing feasibility were respectively above the age of 60 or 70 . The most common exclusion criteria were severe 
Table 4 Setting and study characteristics feasibility

\begin{tabular}{|c|c|c|c|}
\hline Study & Population \& setting & Intervention & Feasibility \\
\hline \multirow[t]{4}{*}{ Brown 2006 [30] } & Intervention $(n=7)$ & \multirow{2}{*}{$\begin{array}{l}\text { Exercise sessions twice a day, } 7 \text { days a week. After discharge } \\
20-30 \text { min walk each day and resistance exercise every other day }\end{array}$} & $\mathrm{n}=605$ admitted, $\mathrm{n}=76$ included \\
\hline & $\begin{array}{l}\text { Mean age } 70.2 \text { years, } \\
0 \% \text { female }\end{array}$ & & $\mathrm{n}=66$ declined participation \\
\hline & Control $(n=2)$ & \multirow{2}{*}{$\begin{array}{l}\text { Usual care which included physical therapy if a consult was } \\
\text { initiated by the physician }\end{array}$} & \\
\hline & $\begin{array}{l}\text { Mean age } 70.2 \text { years, } \\
0 \% \text { female }\end{array}$ & & \\
\hline \multirow[t]{7}{*}{ Mallery 2003 [31] } & Intervention $(\mathrm{n}=19)$ & \multirow{4}{*}{$\begin{array}{l}\text { Usual care plus resistance exercise } 3 \text { times per week, } \\
30-40 \text { min, assisted by a physiotherapist }\end{array}$} & $\mathrm{n}=395$ admitted, $\mathrm{n}=39$ included \\
\hline & Mean age 82.7 years & & Participation 71\%, \\
\hline & 74\% female, GU, UH & & Adherence 63\% \\
\hline & & & No adverse events \\
\hline & Control $(n=20)$ & \multirow{3}{*}{$\begin{array}{l}\text { Usual care plus passive range of motion training } \\
3 \text { times per week, } 30-40 \text { min, assisted by a physiotherapist }\end{array}$} & Participation 96\%, \\
\hline & Mean age 81.4 years & & Adherence 95\% \\
\hline & $45 \%$ female, GU, UH & & No adverse events \\
\hline \multirow[t]{6}{*}{ Nolan 2008 [32] } & Intervention $(\mathrm{n}=196)$ & \multirow{3}{*}{$\begin{array}{l}\text { Participated in the Functional Maintenance Exercise } \\
\text { Program, } 6 \text { times per week, } 30 \text { min }\end{array}$} & $\mathrm{n}=1021$ admitted, $\mathrm{n}=220$ included \\
\hline & Mean age 83.6 years, & & 33 withdrawn \\
\hline & $68 \%$ female, GU, UH & & \\
\hline & Control $(n=24)$ & \multirow[t]{3}{*}{ Usual care with usual physiotherapy } & \\
\hline & Mean age 85.4 years & & \\
\hline & $67 \%$ female, GU, UH & & \\
\hline \multirow[t]{6}{*}{ Laver 2012 [14] } & Intervention $(\mathrm{n}=22)$ & \multirow{3}{*}{$\begin{array}{l}\text { Individual interactive video game program (Wii Fit) } \\
25 \mathrm{~min} / \text { day, } 5 \text { days/week supervised by a physiotherapist }\end{array}$} & $\mathrm{n}=235$ admitted, $\mathrm{n}=44$ included \\
\hline & Mean age 85.2 years & & $90 \%$ adherence rate \\
\hline & $86 \%$ female GU, $\mathrm{ACH}$ & & No adverse events \\
\hline & Control $(n=22)$ & \multirow{3}{*}{$\begin{array}{l}\text { Conventional physiotherapy, matching the patients } \\
\text { abilities and treatment needs } 25 \mathrm{~min} / \text { day, } 5 \text { days/week }\end{array}$} & 91\% adherence rate \\
\hline & Mean age 84.6 years & & 1 adverse event, \\
\hline & 73\% female GU, ACH & & \\
\hline
\end{tabular}

GU=Geriatric Unit; UH=University Hospital.

impairments in physical performance and cognition, requiring palliative care, expected short length of stay, and medical instability. One study specifically targeted frail patients that were at risk of functional decline [32].

Feasibility was measured quantifying patients enrolment, patient adherence to the program and at patient's safety in the context of the exercise program. Nine of the 11 studies found that between the $14 \%$ and $48 \%$ of the admitted patients met the inclusion to be enrolled in the programs, and between 3 and $19 \%$ of the patients were not willing to participate $[14,17,19,22-24,28,31,32]$. In general patients not willing to participate stated that they did not feel like exercising or that they did not believe they could exercise. They felt unwell and/or were scared that exercising would make them feel worse.

In one study [30] only $2 \%$ of all admitted patients were included. Of the 76 patients that met the inclusion criteria only 10 consented to participating while $87 \%$ of the eligible patients refused to take part in the program. In fact none of the included patients were able to start with the exercise program. Reasons for not participating in the exercise program were not of a physical nature.
Instead, patients were discharged before therapy could begin or were unavailable due to diagnostic tests or appointments with healthcare professionals.

Adherence rates were fairly high between the $60 \%$ and $90 \%[14,20,31,32]$. The most common reasons for dropping out of the intervention programs were early discharge, being transferred to intensive or palliative care, being medically unstable, and death [17-19,22,23,28,32].

A final measure of feasibility is patient safety during the exercise program. One feasibility study and six RCT studies included in this review reported on potential side effects such as injuries, accidents, and more specifically, fall incidents, related to participating in the early physical rehabilitation programs $[14,16,18,20,23,28,31]$. None of the studies found any differences in the number of incidents between the exercise groups and usual care groups.

\section{Discussion}

The first aim of this review was to evaluate the effect of early physical rehabilitation programs for geriatric hospitalized patients on physical functioning. A total of 15 
articles, reporting on 13 studies, were included that provided early physical rehabilitation programs in a hospital setting. The present review shows that early physical rehabilitation programs might be beneficial to prevent rapid decline in physical functioning.

A classification could be made between multidisciplinary programs with an exercise component and usual care with an exercise component. Multidisciplinary programs reduced the length of stay for patients in the exercise groups compared to the patients receiving usual care. Additionally, the patients in the multidisciplinary programs were less likely to be discharged to a nursing home or other forms of institutionalized care than patients in the usual care group. The usual care programs with an exercise intervention had the main aim to improve functional outcomes, some of the studies showed indeed an improvement on ADL and physical performance.

The two types of exercise interventions, e.g. multidisciplinary programs with an exercise component and usual care with an exercise component, did not find different results in physical functioning at time of discharge. At time of discharge, results on physical performance were to some extent contradicting, but the majority of the included studies showed that patients in the exercise groups had better ADL and physical performance than patients in the usual care groups, although those results were not always significant. Follow-up results on ADL and physical performance showed that persistent positive effects were mainly found in studies that provided patients with continuous interventions after hospital discharge. The studies that provided patients with only in-hospital exercise interventions found little or no effect on ADL and physical performance at follow-up examinations. These results are in line with other studies that investigate the effects of care in geriatric units and of inpatient rehabilitation for geriatric patients on functional parameters [10,33]. The effects of only in-hospital interventions were clearly positive at time of discharge but were greatly reduced during follow-up. These results suggest that the recovery of patients could further benefit from a community based or in-home intervention programs which build on inhospital programs. Such programs could consist of physical or occupational therapy. In addition, the present review shows in home visits and follow-up telephone calls might be effective for adherence for home-based exercise [20]. Further research is needed to clarify the effects and feasibility of community based and in-home intervention programs in old adults after hospital discharge.

The studies presented in this review included very heterogeneous groups. Half of all studies excluded nursing home patients. Probably, due to the aim of those studies to facilitate discharge home. The studies in this review that targeted frail older patients, patients with increased risk for readmission or patients with a high risk for nursing home admission found positive effects of an exercise program on ADL, length of stay and discharge destination $[20,25,26]$. This result indicates that frail old adults and nursing home patients may benefit from early rehabilitation. Targeting the right population for early physical rehabilitation may be seen as crucial.

The outcomes with regard to physical functioning were measured by a variety of instruments and at different points in time, some of these instruments demonstrated floor and ceiling effects. Floor and ceiling effects could influence the outcomes and distort the results. There were also cases in which information could only be collected with the help of close relatives and caregivers, because relevant information could not be obtained from the patients themselves. The limitations of the current used measurement instruments implies that there might be a need for more sensitive instruments that measure aspects of physical functioning in hospitalized old adults.

Finally, the second aim of this review was to investigate the feasibility of early physical rehabilitation programs for acute ill older patients. The early physical rehabilitation programs must be safe to perform and may not cause high numbers of drop outs. There were four studies identified reporting on the feasibility of inhospital exercise programs [14,30-32]. Additionally, a part of the randomized controlled trials, used to determine the effect on physical functioning, reported also on some feasibility points. Several studies included in this review affirmed the safety of early rehabilitation programs. Patients in the early physical rehabilitation groups were not more often injured, nor did they experience more adverse events or falls than usual care patients. Results on patient recruitment were contradictory and should be interpreted with care due to the limited number of available studies. Adherence rates were high for most studies. Patient as well as the intervention provider's satisfaction was higher when patients were treated with a early physical rehabilitation program instead of usual care [19]. There was one study that encountered difficulties in recruiting patients and was unable to have any patients participate in the exercise program at all [30]. Patients refused to participate or were unavailable at scheduled time of therapy. One of the greatest barriers in the implementation of intervention research, according to the opinion of nurses, was a lack of awareness and knowledge [34]. Equally important in this regard were difficulties in the cooperation of interdisciplinary team members [34]. Education of staff and patients about the safety and the benefits of early physical rehabilitation as well as regular team conferences that improve coordination might help to increase the success rate of intervention programs with regard to participate. 


\section{Limitations}

The total number of included studies in this review is small and the methodological quality for most studies was moderate. Most articles scored particularly low on blinding the assessor, the care provider, and the participant. However, it may be regarded as almost impossible to assure blinding in the context of early physical rehabilitative interventions. One of the major limitations of the included studies lies with the poor descriptions of exercise in early physical rehabilitation programs. Since there is often no clear description on type, duration, frequency, and intensity of exercises that patients receive. Furthermore, because many multidisciplinary programs are focused on outcomes beyond functionality, e.g. early discharge planning, it is difficult to determine what the effect of the exercise component on physical functioning is in this setting. Future research should provide quantification of exercises that older patients perform during their hospital stay, so that there would be a clearer view on the dose-response relationship of physical activity and functional outcomes.

\section{Conclusion}

Early physical rehabilitation programs for acutely hospitalized old adults have the potential to improve physical functioning, and also to prevent patients from being discharged to nursing homes or other forms of institutionalized care. Interventions including a follow-up program after hospital discharge increase the chance of maintaining positive effects on functionality for longer periods of time. Early physical rehabilitation for acutely hospitalized older adults seem to be safe to execute in terms of adverse events such as falls or other injuries, but recruiting the most suitable patients and getting them to participate regularly in the program can be a challenge. Therefore, the commitment and collaboration of staff is of great importance. Further research is needed to quantify the physical activity of patients in early physical rehabilitation programs and to determine the effects and feasibility of community-based and inhome exercise programs.

\section{Competing interests}

The authors declare that they have no proprietary, financial, professional, or other personal competing interests of any nature or kind.

\section{Authors' contribution}

Nienke Kosse and Alisa Dutmer managed the review process and wrote the draft of the full manuscript together with Claudine Lamoth. All authors critically revised the manuscript and approved the final version.

\section{Acknowledgements}

This review is part of the project "Telemedicine \& Personalized Care - project fall prevention" and co-financed, in the context of the INTERREG IV A Program Deutschland-Netherlands, by the European Regional Development Fund (ERDF) and the Ministries of Economic Affairs of the Netherlands and the German states of Nordrhein-Westfalen and Niedersachsen.

\section{Author details}

${ }^{1}$ University of Groningen, University Medical Center Groningen, Center for Human Movement Sciences, Groningen, The Netherlands. ${ }^{2}$ Geriatrics Center Oldenburg, Carl von Ossietzky Universität Oldenburg, Oldenburg, Germany.

Received: 15 May 2013 Accepted: 8 October 2013

Published: 10 October 2013

\section{References}

1. OECD: Health at a Glance: Europe 2012. OECD Publishing; 2012 [http://dx.doi. org/10.1787/9789264183869-en].

2. Creditor MC: Hazards of Hospitalization of the Elderly. Ann Intern Med 1993, 118(3):219.

3. Sager MA, Franke T, Inouye SK, Landefeld CS, Morgan TM, Rudberg MA, Sebens $\mathrm{H}$, Winograd $\mathrm{CH}$ : Functional outcomes of acute medical illness and hospitalization in older persons. Arch Intern Med 1996, 156(6):645-652.

4. Covinsky KE, Palmer RM, Fortinsky RH, Counsell SR, Stewart AL, Kresevic D, Burant CJ, Landefeld CS: Loss of independence in activities of daily living in older adults hospitalized with medical illnesses: increased vulnerability with age. J Am Geriatr Soc 2003, 51(4):451-458.

5. McVey LJ, Becker PM, Saltz CC, Feussner JR, Cohen HJ: Effect of a geriatric consultation team on functional status of elderly hospitalized patients. Ann Intern Med 1989, 110(1):79.

6. Fortinsky RH, Covinsky KE, Palmer RM, Landefeld CS: Effects of functional status changes before and during hospitalization on nursing home admission of older adults. J Gerontol A Biol Sci Med Sci 1999, 54(10):M521-M526.

7. Rudberg MA, Sager MA, Zhang J: Risk factors for nursing home use after hospitalization for medical illness. J Gerontol A Biol Sci Med Sci 1996, 51(5): M189-M194.

8. Kortebein P, Symons TB, Ferrando A, Paddon-Jones D, Ronsen O, Protas E, Conger S, Lombeida J, Wolfe R, Evans WJ: Functional impact of 10 days of bed rest in healthy older adults. J Gerontol A Biol Sci Med Sci 2008, 63(10):1076-1081.

9. Mendez-Tellez PA, Nusr R, Feldman D, Needham DM: Early physical rehabilitation in the ICU: a review for the neurohospitalist. The Neurohospitalist 2012, 2(3):96-105.

10. Baztan JJ, Suarez-Garcia FM, Lopez-Arrieta J, Rodriguez-Manas L, Rodriguez-Artalejo F: Effectiveness of acute geriatric units on functional decline, living at home, and case fatality among older patients admitted to hospital for acute medical disorders: meta-analysis. BMJ 2009, 338:b50.

11. de Morton NA, Keating $J$, Jeffs $K$ : The effect of exercise on outcomes for older acute medical inpatients compared with control or alternative treatments: a systematic review of randomized controlled trials. Clin Rehabil 2007, 21(1):3-16.

12. Verhagen $A P$, de Vet $H C$, de Bie RA, Kessels AG, Boers M, Bouter LM, Knipschild PG: The Delphi list: a criteria list for quality assessment of randomized clinical trials for conducting systematic reviews developed by Delphi consensus. J Clin Epidemiol 1998, 51(12):1235-1241.

13. Olivo SA, Macedo LG, Gadotti IC, Fuentes J, Stanton T, Magee DJ: Scales to assess the quality of randomized controlled trials: a systematic review. Phys Ther 2008, 88(2):156-175.

14. Laver K, George S, Ratcliffe J, Quinn S, Whitehead C, Davies O, Crotty M: Use of an interactive video gaming program compared with conventional physiotherapy for hospitalised older adults: a feasibility trial. Disabil Rehabil 2012, 34(21):1802-1808.

15. Liberati A, Altman DG, Tetzlaff J, Mulrow C, Gotzsche PC, loannidis JP, Clarke M, Devereaux PJ, Kleijnen J, Moher D: The PRISMA statement for reporting systematic reviews and meta-analyses of studies that evaluate health care interventions: explanation and elaboration. J Clin Epidemiol 2009, 62(10):e1-e34.

16. Abizanda $P$, Leon M, Dominguez-Martin L, Lozano-Berrio V, Romero L, Luengo C, Sanchez-Jurado PM, Martin-Sebastia E: Effects of a short-term occupational therapy intervention in an acute geriatric unit. A randomized clinical trial. Maturitas 2011, 69(3):273-278.

17. Asplund K, Gustafson Y, Jacobsson C, Bucht G, Wahlin A, Peterson J, Blom JO, Angquist KA: Geriatric-based versus general wards for older acute medical patients: a randomized comparison of outcomes and use of resources. J Am Geriatr Soc 2000, 48(11):1381-1388.

18. Blanc-Bisson C, Dechamps A, Gouspillou G, Dehail P, Bourdel-Marchasson I: $A$ randomized controlled trial on early physiotherapy intervention versus 
usual care in acute care unit for elderly: potential benefits in light of dietary intakes. J Nutr Health Aging 2008, 12(6):395-399.

19. Counsell SR, Holder CM, Liebenauer LL, Palmer RM, Fortinsky RH, Kresevic DM, Quinn LM, Allen KR, Covinsky KE, Landefeld CS: Effects of a multicomponent intervention on functional outcomes and process of care in hospitalized older patients: a randomized controlled trial of Acute Care for Elders (ACE) in a community hospital. J Am Geriatr Soc 2000, 48(12):1572-1581.

20. Courtney MD, Edwards HE, Chang AM, Parker AW, Finlayson K, Bradbury C, Nielsen Z: Improved functional ability and independence in activities of daily living for older adults at high risk of hospital readmission: a randomized controlled trial. J Eval Clin Pract 2012, 18(1):128-134.

21. Courtney M, Edwards H, Chang A, Parker A, Finlayson K, Hamilton K: Fewer emergency readmissions and better quality of life for older adults at risk of hospital readmission: a randomized controlled trial to determine the effectiveness of a 24-week exercise and telephone follow-up program. J Am Geriatr Soc 2009, 57(3):395-402.

22. de Morton NA, Keating JL, Berlowitz DJ, Jackson B, Lim WK: Additional exercise does not change hospital or patient outcomes in older medical patients: a controlled clinical trial. Aust J Physiother 2007, 53(2):105-111.

23. Jones $C T$, Lowe AJ, MacGregor L, Brand CA, Tweddle N, Russell DM:

A randomised controlled trial of an exercise intervention to reduce functional decline and health service utilisation in the hospitalised elderly. Australas J Ageing 2006, 25(3):126-133.

24. Landefeld CS, Palmer RM, Kresevic DM, Fortinsky RH, Kowal J:

A randomized trial of care in a hospital medical unit especially designed to improve the functional outcomes of acutely ill older patients. N Engl J Med 1995, 332(20):1338-1344.

25. Nikolaus T, Specht-Leible N, Bach M, Oster P, Schlierf G: A randomized trial of comprehensive geriatric assessment and home intervention in the care of hospitalized patients. Age Ageing 1999, 28(6):543-550.

26. Saltvedt I, Jordhoy M, Opdahl Mo ES, Fayers P, Kaasa S, Sletvold O: Randomised trial of in-hospital geriatric intervention: impact on function and morale. Gerontology 2006, 52(4):223-230.

27. Saltvedt I, Mo ES, Fayers P, Kaasa S, Sletvold O: Reduced mortality in treating acutely sick, frail older patients in a geriatric evaluation and management unit. A prospective randomized trial. J Am Geriatr Soc 2002, 50(5):792-798.

28. Siebens H, Aronow H, Edwards D, Ghasemi Z: A randomized controlled trial of exercise to improve outcomes of acute hospitalization in older adults. J Am Geriatr Soc 2000, 48(12):1545-1552.

29. Slaets JP, Kauffmann RH, Duivenvoorden HJ, Pelemans W, Schudel WJ: A randomized trial of geriatric liaison intervention in elderly medical inpatients. Psychosom Med 1997, 59(6):585-591.

30. Brown CJ, Peel C, Bamman MM, Allman RM: Exercise program implementation proves not feasible during acute care hospitalization. J Rehabil Res Dev 2006, 43(7):939-946.

31. Mallery LH, MacDonald EA, Hubley-Kozey CL, Earl ME, Rockwood K, Macknight $C$ : The feasibility of performing resistance exercise with acutely ill hospitalized older adults. BMC Geriatr 2003, 3:3.

32. Nolan J, Thomas S: Targeted individual exercise programmes for older medical patients are feasible, and may change hospital and patient outcomes: a service improvement project. BMC Health Serv Res 2008 $8: 250$.

33. Bachmann S, Finger C, Huss A, Egger M, Stuck AE, Clough-Gorr KM: Inpatient rehabilitation specifically designed for geriatric patients: systematic review and meta-analysis of randomised controlled trials. BMJ 2010, 340:C1718.

34. Hutchinson AM, Johnston L: Bridging the divide: a survey of nurses opinions regarding barriers to, and facilitators of, research utilization in the practice setting. J Clin Nurs 2004, 13(3):304-315.

doi:10.1186/1471-2318-13-107

Cite this article as: Kosse et al:: Effectiveness and feasibility of early physical rehabilitation programs for geriatric hospitalized patients: a systematic review. BMC Geriatrics 2013 13:107.

\section{Submit your next manuscript to BioMed Central and take full advantage of:}

- Convenient online submission

- Thorough peer review

- No space constraints or color figure charges

- Immediate publication on acceptance

- Inclusion in PubMed, CAS, Scopus and Google Scholar

- Research which is freely available for redistribution

Submit your manuscript at www.biomedcentral.com/submit
Ciomed Central 